\title{
Dietary Anthocyanins- Smart Molecules with Chemo Preventive and Chemotherapeutic Activity
}

\author{
K V Surangi Dharmawansa* \\ Department of Plant, Food, and Environmental Sciences, Faculty of Agriculture, Dalhousie University, Canada
}

Submission: September 20, 2020; Published: October 19, 2020

${ }^{*}$ Corresponding author: K V Surangi Dharmawansa, Department of Plant, Food, and Environmental Sciences, Faculty of Agriculture, Dalhousie University, Truro, NS, Canada

\begin{abstract}
Cancer is the second leading cause of death in global and has a major impact on human wellbeing. Recently, dietary interventions in cancer prevention and use of plant secondary metabolites in cancer therapies have been received a considerable attention. Anthocyanin, a group of flavonoids have investigated extensively for its chemopreventive and chemotherapeutic activity against the several types of cancers. Anthocyanin possess strong antioxidant activity. In addition to that, anthocyanin have shown the anti-inflammatory, anti-proliferative, apoptotic, and antitumorigenic properties in both in vitro and in vivo. This mini review summarizes the potential molecular mechanisms and anti-cancer activity of anthocyanin towards the breast, lung, and colorectal cancer.
\end{abstract}

Keywords: Anthocyanin; Chemoprevention; Chemotherapy; Breast cancer; Lung cancer; Colorectal cancer

\section{Anti-Cancer Mechanisms}

Anthocyanins, categorized under the plant flavonoids, are derived from the basic structure of anthocyanidins which consist of two aromatic rings and one heterocyclic ring having a C6C3-C6 basic skeleton [1].To date, more than 635 of anthocyanin compounds have been identified and they differ in the number of hydroxylated groups, the type and number of conjugated sugars and the presence or absence of acyl groups. Among them, the most common anthocyanins found in nature are cyanidin, delphinidin, pelargonidin, peonidin, petunidin and, malvidin [2]. Anthocyanins are widely distributed in leaves, flowers, roots, fruits, and grains of many colorful fruits and vegetables [3]. Presence of anthocyanin exhibits via the different intensities of blue, purple, violet and red shades in the food crops. Once administrated orally, anthocyanins are readily absorbed in stomach and undergo first phase metabolism and become available in the circulatory system within few minutes [4]. However, anthocyanin has a very low systemic bioavailability [5].

Hence, studies are being conducted to improve the gastric survivability and bioavailability of anthocyanins in human body. Anthocyanins which were not absorbed in the stomach, then passed down to the small intestine and, metabolized in to glucuronidate, sulphate or methylate derivatives and quickly absorb to the small intestine [6]. Unabsorbed, intact anthocyanins are then reached the colon where it subjected to spontaneous metabolism or metabolism mediated by the action of gut microbes and results compounds with structural modifications with unique anticancer properties [5]. Anthocyanin or its metabolites exhibit anticancer properties for their antioxidant activity, antiinflammatory activity, functions in genome stabilization, activities in the modulation of cell signaling, suppression of cancer cell proliferation and interactions with gut micro flora. Apart from that, anthocyanins have been identified as the potent sources of stimulating the expression of tumor suppressor genes and downregulating the pro-oncogenic signals as well as inducing the apoptosis.

Being one of the potent antioxidants, anthocyanin scavenges free radicals and reduce the DNA damage, gene mutations, thus prevents tumor development [7]. Excessive chronic inflammation is associated with onset and progression of cancers. According to the available literature, it has found that anthocyanins are also act as anti-inflammatory agents [8]. Anthocyanin-rich wild blueberry extract reduced the inflammation in human Caco-2 cells by decreasing the activation of NF-kB [9]. Moreover, anthocyanin have been evidenced in inhibiting the cell proliferation via 
arresting the cell cycle at different mediated phases. The cell cycle is blocked by exerting effects on cell cycle regulatory proteins like p53, p21, p27, and cyclin D1 cyclin A and cyclin B [10]. The HT-29 human colon cancer cells treated with anthocyanin, showed cell cycle blockage at G1/G0 and G2/M phases [11]. Further, increased levels of expression in p21 and p27 genes and decreased levels of expression in cyclin A and B genes were observed. Anthocyanins also involved in two pathways of cell apoptosis namely, the extrinsic pathway of activation of death receptors on the cell surface and intrinsic mitochondria-mediated pathway.

In the former pathway, anthocyanins modulate the expression of first apoptosis signal (FAS) and first apoptosis signal ligand (FASL) death receptors located on the tumor cell surface. In the latter pathway, anthocyanin increases the mitochondrion membrane potential and facilitate the cytochrome c removal from the cell and modulate caspase-dependent apoptotic proteins [10]. Anthocyanin-rich purple-flesh potato induced apoptosis in colon cancer stem cells in a p53-independent manner in-vitro and suppressed the tumor incidence and the number of colon cancer stem cells by interfering with the Wnt- $\beta /$ Catenin pathway in-vivo [12]. Same research group evaluated the effect of anthocyaninrich (including glucosides of delphinidin, cyanidin, petunidin, peonidin, and malvidin) java plum fruit extract on HCT-116 colon cancer cell line and colon cancer stem cells. Interestingly, anthocyanin extract suppressed cell proliferation and apoptosis in both cells and stemness in colon cancer stem cells [13]. In addition to those promising anti-cancer effects, anthocyanin also engaged in suppressing the activity of matrix metalloproteinases (MMP) 2 and 9.

MMPs are a large family of about 25-Zinc-dependent endopeptidases and can degrade components in the extracellular matrix and extensively involved in cellular damage which ultimately leads to cancer metastasis [14]. As evidenced by invitro and in-vivo studies, anthocyanin is potent in inhibiting the expression of MMP-2 and MMP-9 via down regulating the reactive oxygen species [15]. Even though the exact mechanism is unclear, dysbiosis of gut microflora which associates with increased levels of Helicobacter pylori, Streptococcus gallolyticus, Enterococcus faecalis and Streptococcus bovis etc. has being identified as a major influence of gastrointestinal cancers. However, anthocyanins and their metabolites (phloroglucinol derivatives, hydroxyphenyiacetic acid, benzoic acid, demethylated compounds of phenolic acids like syringic acid, transitorily acid pyrogallol, photocatecheuic acid, 2,4,6-trihydroxybalsaldehyde and gallic acid) have interestingly re-modulated the dysbiosis in the gut and thereby prevent the gastrointestinal cancers [16].

\section{Breast Cancer}

Pure anthocyanin and anthocyanin rich extracts have been exhibited anti-cell proliferative effect towards different types breast cancer cells $[17,18]$. For an example, anthocyanin extracted from Mixteco blue corn inhibited the cell proliferation in triple negative breast cancer cells by arresting the cell cycle at G1 phase
[18]. In another study, anthocyanin extracted from strawberry was potent in inhibiting the cell proliferation in two murine breast cancer cell lines [17]. Further, strawberry anthocyanin induced the cell apoptosis via increasing the cellular oxidative stress. Anthocyanin has been investigated for its anti-angiogenesis activity as well. Angiogenesis plays a vital role in breast cancer progression. Cyanidin-3-0-glucoside $\left(\mathrm{C}_{3} \mathrm{G}\right)$, one of the most widely distributed anthocyanins in edible fruits, showed attenuation in breast cancer induced angiogenesis via inhibiting the vascular endothelial growth factor (VEGF).

Further, $\mathrm{C}_{3} \mathrm{G}$ inhibited the expression of signal transducer and activator of transcription 3 (STAT3) which involves in transcriptional activation of VEGF [19]. Overexpression of human epidermal growth factor receptor 2 (HER2) drives the biology of $30 \%$ of breast cancer cases which associates with RAS/RAF/MAPK pathway. Anthocyanin extracted from black rice inhibited cell migration and invasion, suppressed the activation of mitogen-activated protein kinase (RAF), mitogenactivated protein kinase (MEK), and c-Jun N-terminal kinase (JNK), and downregulated the secretion of MMP2 and MMP9 which contributes to the antimetastatic activity [20]. In another study, black rice anthocyanin decreased the migration distance of the HER-2-positive human breast cancer cells, MDA-MB-453, by $37 \%$ [21]. Further, anthocyanin reduced the number of invading MDA-MB-453 cells and showed inhibitory effect on epithelialmesenchymal transition. Moreover, anthocyanin increased the expression of the epithelial marker, E-cadherin, and decreased the expression of the mesenchymal markers, fibronectin, and vimentin, in the MDA-MB-453 cells.

\section{Lung Cancer}

Dietary interventions in lung cancer prevention and treatment have been gained significant attention during the past decade. Among them use of anthocyanin in lung cancer prevention has investigated extensively. Delphinidin inhibited the VEGF expression at protein and mRNA levels in A549 lung cancer cells and thereby reduced the angiogenesis [22]. Delphinidin also associated with reducing the expression of hypoxia-inducible factor (HIF)- $1 \alpha$, which is a transcription factor of VEGF. In another study which used the same lung cancer cell model, anthocyanin from fruits of Vitis coignetiae pulliat exhibited inhibition of cancer cell proliferation, invasion and angiogenesis [23]. Further, anthocyanin suppressed cancer migration, and invasion by supressing MMP-2 and MMP-9 expression and suppressed the different types of proteins involved in cancer proliferation (COX-2, C-myc, cyclin D1), migration and invasion (MMP-2, MMP-9), antiapoptosis (XIAP, and c-IAP2), adhesion and angiogenesis (ICAM-1, VEGF).

Mitogen-activated protein kinase (MAPK) family proteins play an important role in complex cellular programs like proliferation, differentiation, development, transformation, and apoptosis [24]. Peonidine-3-glucoside (P3G) is evidenced for attenuating the phosphorylation of extracellular signal-regulated kinase 
(ERK)1/2, a member of MAPK family in lung cancer carcinoma [25]. ERK1/2 is involved in cancer metastasis via upregulating the MMPs. The synergistic effect of anthocyanin mixtures has been investigated by Kausar et al. [26] on the non-small-cell lung cancer cells (NSCLC). According to the findings, a mixture of blueberry, bilberry and Indian blackberry anthocyanins were potent in inhibiting the growth of NSCLC H1299 and A549 lung cancer cells via induction of cell-cycle arrest, apoptosis and suppression of NSCLC cell invasion and migration. Similar results were observed in a study conducted with mulberry anthocyanins, $\mathrm{C}_{3} \mathrm{G}$ and, cyanidin-3-rutinoside in A549 lung carcinoma cells in which it decreased the expressions of MMP-2 and urokinase-plasminogen activator (u-PA) in a dose-dependent manner and enhanced the expression of tissue inhibitor of matrix matalloprotinase-2 (TIMP2) and plasminogen activator inhibitor (PAI) [27].

\section{Colorectal Cancer}

There are many studies have been done to access the effect of anthocyanin on CRC prevention both in-vitro and in-vivo. Moreover, recent meta-analysis revealed the inverse association between anthocyanin consumption and the colorectal cancer incidence [28]. As Fernández et al. [29] revealed, Rattus norvegicus $\left(\mathrm{F}_{344}\right)$ rats who received two doses of (AOM) $(10 \mathrm{mg} / \mathrm{kg})$ and fed with functional sausages containing $0.1 \%(\mathrm{w} / \mathrm{w})$ of anthocyanin from a mixture of dehydrated blackberries and strawberries for 20 weeks displayed less tumor number, size, and progression. Another study has shown the effectiveness of anthocyanin-rich extract (ARE) from bilberries on colorectal tumor development by administration of AOM, dextran sodium sulfate (DSS) in mouse. Mice received $10 \%$ anthocyanin showed significantly less reduced colon length indicating less inflammation and less mean tumor numbers [30]. In addition to that, purple sweet potato (PSP), red cabbage and purple corn color (PCC), which are rich in anthocyanin, have also been shown to inhibit chemically-induced carcinogenesis in male $\mathrm{F}_{344} /$ DuCrj rats, initially treated with 1,2-dimethylhydrazine (DMH) [31]. There was a significant reduction in the incidence and multiplicity of colonic adenomas and adenocarcinomas of all dosed groups with compared to control rats. In concern to the epidemiological experiments, Before and after oral consumption of black raspberry powder as $60 \mathrm{~g}$ /day up to 19 weeks, potent in upregulating the tumor suppressor genes in 20 colorectal cancer patients [32].

\section{Conclusion}

Dietary anthocyanins are absorbed to the body tissues at main three sites in the gastrointestinal tract: at stomach, small intestine and colon. Despite the amount consumed or the type of anthocyanin consumed, bioavailability of anthocyanin is very low. Majority of the anthocyanins passed down to the colon and subjected to microbial metabolism while resulting phenolic acids, their derivatives, and aldehydes. Unabsorbed or intact anthocyanin then excreted through urine, feces, and exhaled air. Anthocyanin possesses anti-cancer properties mainly due to their strong electron donor ability, achieved via the presence of several hydroxyl groups attached to its structure. Anthocyanin demonstrated several mechanisms of actions in the prevention of cancer. Scavenging of ROS, RON and free radicals due to their antioxidant activity, induction of tumor cell apoptosis, control of expression and secretion of inflammatory factors, retardation of cell proliferation via interfering with cell cycle and suppression of matrix metalloproteinase are main anti-cancer mechanisms performed by anthocyanins and their metabolites towards the cancer chemoprevention. Since anthocyanins are readily available and rich in colorful fruits and vegetables, they can be identified as a promising source of cancer prevention. But as for the future directions, pharmacological activity of anthocyanin/ or their derivatives/or metabolites need to be evaluated via epidemiological studies. Moreover, to increase the bioavailability of those phytochemicals, efficient technologies like encapsulation can be utilized.

\section{References}

1. Clifford M N (2000) Anthocyanins-nature, occurrence and dietary burden. J Sci Food Agric 80: 1063-1072.

2. Castañeda Ovando A, Pacheco Hernández M de L, Páez Hernández M E, Rodríguez J A, Galán Vidal C A (2009) Chemical studies of anthocyanins: A review. Food Chem 113: 859-871.

3. Khoo H E, Azlan A, Tang S T, Lim S M (2017) Anthocyanidins and anthocyanins: Colored pigments as food, pharmaceutical ingredients, and the potential health benefits. Food Nutr Res 61: 1361779.

4. Liu Y, Zhang D, Wu Y, Wang D, Wei Y, et al. (2014) Stability and absorption of anthocyanins from blueberries subjected to a simulated digestion process. Int J Food Sci Nutr 65: 440-448.

5. Fernandes I, de Freitas V, Mateus N (2014) Anthocyanins and human health: How gastric absorption may influence acute human physiology. Nutr Aging 2: 1-14.

6. Fernandes I, Faria A, Calhau C, de Freitas V, Mateus N (2014) Bioavailability of anthocyanins and derivatives. J Funct Foods 7: 54-66.

7. Yi L, Chen C ye, Jin X, Mi M tian, Yu B, et al. (2010) Structural requirements of anthocyanins in relation to inhibition of endothelial injury induced by oxidized low-density lipoprotein and correlation with radical scavenging activity. FEBS Lett 584: 583-590.

8. Esposito D, Chen A, Grace M H, Komarnytsky S, Lila M A (2014) Inhibitory effects of wild blueberry anthocyanins and other flavonoids on biomarkers of acute and chronic inflammation in vitro. J Agric Food Chem 62: 7022-7028.

9. Li D, Wang P, Luo Y, Zhao M, Chen F (2017) Health benefits of anthocyanins and molecular mechanisms: Update from recent decade. Crit Rev Food Sci Nutr 57: 1729-1741.

10. Wang Li shu, Stoner G D (2008) Anthocyanins and their role in cancer prevention. Cancer Lett 269: 281-290.

11. Hsu C P P, Shih Y T T, Lin B R R, Chiu C F F, Lin C C C (2012) Inhibitory effect and mechanisms of an anthocyanins- and anthocyanidins-rich extract from purple-shoot tea on colorectal carcinoma cell proliferation. J Agric Food Chem 60: 3686-3692.

12. Charepalli V, Reddivari L, Radhakrishnan S, Vadde R, Agarwal R, et al. (2015) Anthocyanin-containing, purple-fleshed potatoes suppress colon tumorigenesis via elimination of colon cancer stem cells. J Nutr Biochem 26: 1641-1649. 
13. Charepalli V, Reddivari L, Vadde R, Walia S, Radhakrishnan S, et al. (2016) Eugenia jambolana (Java Plum) Fruit Extract Exhibits AntiCancer Activity against Early Stage Human HCT-116 Colon Cancer Cells and Colon Cancer Stem Cells. Cancers (Basel) 8: 29.

14. Alam M N, Almoyad M Huq F (2018) Polyphenols in Colorectal Cancer: Current State of Knowledge including Clinical Trials and Molecular Mechanism of Action. Biomed Res 2018.

15. Kim S J, Park Y S, Paik H D, Chang H I (2011) Effect of anthocyanins on expression of matrix metalloproteinase-2 in naproxen-induced gastric ulcers. Br J Nutr 106: 1792-1801.

16. Jahani Sherafat S, Alebouyeh M, Moghim S, Amoli H A, Ghasemian Safaei H (2018) Role of gut microbiota in the pathogenesis of colorectal cancer; A review article. Gastroenterol Hepatol from Bed to Bench 11: 101-109.

17. Mazzoni L, Giampieri F, Alvarez Suarez J M, Gasparrini M, Mezzetti B, et al. (2019) Isolation of strawberry anthocyanin-rich fractions and their mechanisms of action against murine breast cancer cell lines. Food Funct 10: 7103-7120.

18. Herrera Sotero M Y, Cruz Hernández C D, Oliart Ros R M, Chávez Servia J L, Guzmán Gerónimo R I, et al. (2020) Anthocyanins of Blue Corn and Tortilla Arrest Cell Cycle and Induce Apoptosis on Breast and Prostate Cancer Cells. Nutr Cancer 72: 768-777.

19. Ma X, Ning S (2019) Cyanidin-3-glucoside attenuates the angiogenesis of breast cancer via inhibiting STAT3/VEGF pathway. Phyther Res 33: 81-89.

20. Chen X Y, Zhou J, Luo L P, Han B, Li F, et al. (2015) Black Rice Anthocyanins Suppress Metastasis of Breast Cancer Cells by Targeting RAS/RAF/MAPK Pathway. Biomed Res Int 2015.

21. Zhou J, Zhu Y F, Chen X Y, Han B, Li F, et al. (2017) Black rice-derived anthocyanins inhibit HER-2-positive breast cancer epithelialmesenchymal transition-mediated metastasis in vitro by suppressing FAK signaling. Int J Mol Med 40: 1649-1656.

22. Kim M H, Jeong Y J, Cho H J, Hoe H S, Park K K, et al. (2017) Delphinidin inhibits angiogenesis through the suppression of HIF- $1 \alpha$ and VEGF expression in A549 lung cancer cells. Oncol Rep 37: 777-784.
23. Lu J N, Panchanathan R, Lee W S, Kim H J, Kim D H, et al. (2017) Anthocyanins from the fruit of vitis coignetiae pulliat inhibit TNFAugmented cancer proliferation, migration, and invasion in A549 cells. Asian Pacific J Cancer Prev 18: 2919-2923.

24. Wei Z, Liu H T (2002) MAPK signal pathways in the regulation of cell proliferation in mammalian cells. Cell Res 12: 9-18.

25. Ho M L, Chen P N, Chu S C, Kuo D Y, Kuo W H, et al. (2010) Peonidin 3 -glucoside inhibits lung cancer metastasis by downregulation of proteinases activities and MAPK pathway. Nutr Cancer 62: 505-516.

26. Kausar, H, Jeyabalan, J, Aqil, F, Chabba, D, Sidana J, et al. (2012) Berry anthocyanidins synergistically suppress growth and invasive potential of human non-small-cell lung cancer cells. Cancer Lett 325: 54-62.

27. Chen P N, Chu S C, Chiou H L, Kuo W H, Chiang C L, et al. (2006) Mulberry anthocyanins, cyanidin 3-rutinoside and cyanidin 3-glucoside, exhibited an inhibitory effect on the migration and invasion of a human lung cancer cell line. Cancer Lett 235: 248-259.

28. Wang X, Yang D Y, Yang L Q, Zhao W Z, Cai L Y, et al. (2019) Anthocyanin Consumption and Risk of Colorectal Cancer: A Meta-Analysis of Observational Studies. J Am Coll Nutr 38: 470-477.

29. Fernández J, García L, Monte J, Villar C J, Lombó, F (2018) Functional Anthocyanin-Rich Sausages Diminish Colorectal Cancer in an Animal Model and Reduce Pro-Inflammatory Bacteria in the Intestinal Microbiota. Genes (Basel) 9: 133.

30. Lippert E, Ruemmele P, Obermeier F, Goelder S, Kunst C, et al. (2017) Anthocyanins prevent colorectal cancer development in a mouse model. Digestion 95: 275-280.

31. Thomasset S, Berry D P, Cai H, West K, Marczylo T H, et al. (2009) Pilot study of oral anthocyanins for colorectal cancer chemoprevention. Cancer Prev Res 2: 625-633.

32. Mentor Marcel R A, Bobe G Sardo, C Wang L S, Kuo C T, et al. (2012) Plasma cytokines as potential response indicators to dietary freezedried black raspberries in colorectal cancer patients. Nutr Cancer 64 820-825.

\begin{tabular}{l} 
Your next submission with Juniper Publishers \\
will reach you the below assets \\
- Quality Editorial service \\
- Swift Peer Review \\
- Reprints availability \\
- E-prints Service \\
- Manuscript Podcast for convenient understanding \\
- Global attai nment for your research \\
- Manuscript accessibility in different formats \\
( Pdf, E-pub, Full Text, Audio) \\
- Unceasing customer service \\
Track the below URL for one-step submission \\
https://juniperpublishers.com/online-submission.php \\
\hline
\end{tabular}

